\title{
Segmentation of T1-MRI of the Human Cortex Using a 3D Grey-Level Morphology Approach
}

\author{
Roger Hult ${ }^{1,2}$ \\ ${ }^{1}$ Centre for Image Analysis, Uppsala University, SE-752 37 Uppsala, Sweden, \\ ${ }^{2}$ Dept. Clinical Neuroscience, Human Brain Informatics \\ Karolinska Institutet, SE-171 76 Stockholm, Sweden, \\ rogerh@cb.uu.se, \\ http://www.cb.uu.se, http://www.hubin.org
}

\begin{abstract}
In this paper, an algorithm for fully automatic segmentation of the cortex from T1-weighted transversal, coronal, or sagittal MRI data is presented. The segmentation algorithm uses a histogram-based method to find accurate threshold values. There are four initial masks created: first two thresholded masks from the original volume, providing background and brain tissue; then a third mask thresholded from a 3D grey-level eroded version of the volume, providing brain tissue; and lastly a fourth mask thresholded from a 3D grey-level dilated version of the volume, providing surrounding fat. On the start slice of these masks binary morphological operations and logical operations are used; then the rest of the slices are segmented using information from the previous slice combined with the other masks. Information from earlier slices is propagated to keep the segmented volume from leaking into non-brain tissue.
\end{abstract}

\section{Introduction}

Magnetic resonance imaging (MRI) provides detailed information of the anatomy of the object that is examined. In a T1-weighted image, fat appears as bright, i.e. relatively short $\mathrm{T} 1$ values; white and grey matter are somewhat darker; fluids and bone tissue appear even darker, i.e. long T1 values. The process of segmenting the cortex is required before analysis and visualisation [4]. There are several segmentation methods for MR brain images, but those methods do not use grey-level morphology.

Several methods are based on statistical approaches [6] as well as methods using artificial neural networks [16], [17]. These methods will not work on a single T1-weighted MRI-data-set, but are multi-spectral approaches that require at least an additional T2-weighted MRI-data-set. Some statistical methods use only one MRI scan, by examining statistical characteristics of the histogram [9]. There are methods that use only T1-weighted MRIs to classify grey and white matter applying a histogram approach [12]. Methods that find thresholds using an iterative approach [15] also exist. These methods also use binary morphology to break connections to non-brain tissue. 
In the method discussed in this paper, the main improvements compared to earlier work [5], [3] are that sagittal and coronal MRI are special cases and that most of the bright non-brain tissues and background are removed. For an example of a 3D visualisation of a segmented brain, segmented with the algorithm, see Fig. 1.

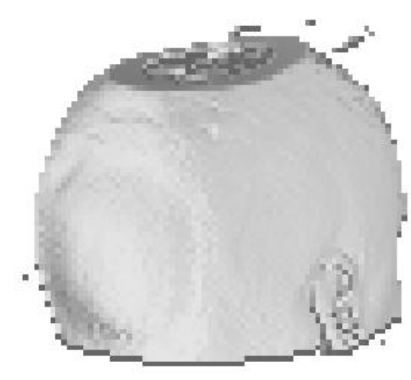

(a)

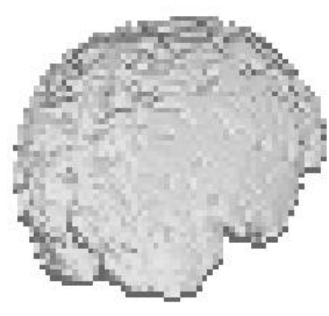

(b)

Fig. 1. A segmented head viewed from behind. The data set used is of very low resolution, $79 \times 95$ with 68 slices, but the algorithm is robust enough to segment the data. a) Original data. b) Segmented cortex.

\section{Methods}

\subsection{Overview}

The method is based on multiple-level thresholding and grey-level morphology combined with anatomical knowledge and binary morphology. A histogrambased method is used to select the threshold intervals. The different kinds of tissues found are brain matter (white matter, grey matter), dark tissue types (fluids, bone, background) and bright tissue types (fat). The thresholding is performed on kernel density estimates (continuous histogram (KDE)) [7], [11], [10]. From this continuous histogram the second derivate is calculated and the four greatest maxima are selected and sorted. See Fig. 2 for an example of how a histogram and the continuous histogram with the second derivate may look. The interval from the lowest to the second maximum then corresponds to CSF and bone, and anything below the first threshold is air. Grey matter is approximately the second to third threshold and white matter is the third to fourth threshold, see Fig. 3a). Surrounding tissues (and sometimes some internal structures in the brain) are above the fourth threshold. Either the complete histogram of the volume or the histogram from the start slice can be used.

In addition to the original MRI-data, called OrgImage, two volumes are calculated. The original MRI-data is grey-level-eroded [13], [14], [1], [2] using a $3 \times 3 \times 3$ structure element; this new volume is called MinImage. The original MRI-data is also grey-level-dilated using a $3 \times 3 \times 3$ structure element; this 


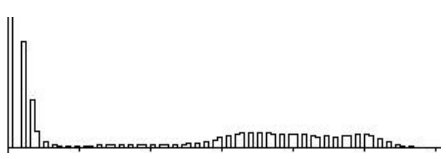

(a)

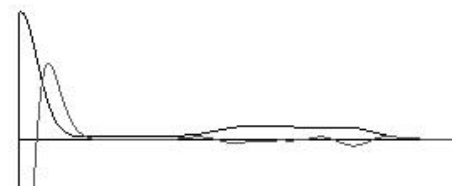

(b)

Fig. 2. a) Grey-level histogram of a mid slice in a 3D MRI data set. b) KDE and second derivate plot of the same slice.

volume is called MaxImage. These images are used to facilitate determining the cortex, by eliminating false connectivities to surrounding tissues. The MinImage is used to make wider gaps between the cortex and those tissues that are not to be included, see Fig. 3b). To find surrounding fat the MaxImage is used, see Fig. 3c). In the grey-level eroded volume the background is below the second $\max$ found from the second derivate of the continuous histogram. In the greylevel dilated volume the surrounding tissue is above the third max found in the same manner as in the previous case.

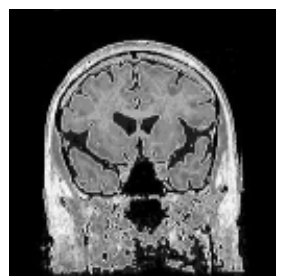

(a)

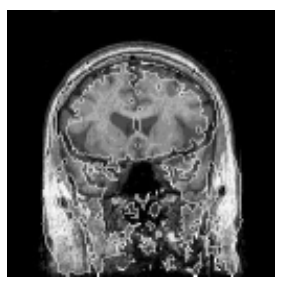

(a)

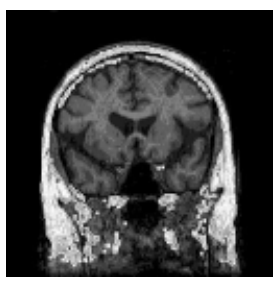

(a)

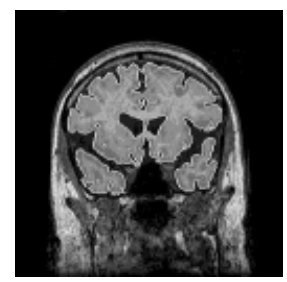

(c)

Fig. 3. a) Brain tissue. b) Brain tissue after grey-level erosion. c) Fat tissue after grey-level dilation. d) Segmented brain.

As of now, there are three versions of the algorithm. The first implementation only handled transaxial MRI data, but now special cases for sagittal and coronal MRI data has been developed. In the transaxial case a slice in the middle of a brain is selected as the start slice, see Fig. 4a). The foreground and background are determined and the information of previous segmentation (both of brain tissue and non-brain tissue) is then applied on the following slices. In the sagittal case, two slices are determined, one in the left hemisphere and one in the right hemisphere, see Fig. 4b). These are chosen as starting slices and are selected from a bounding-box of thresholded tissue to assure that approximately the same slices from the brain are selected. The distance to this slice is one fourth of the distance from the outermost part towards the outermost part of the other side. As in the transaxial case, the information from previously segmented slices propagates throughout the traversing of the volume. In the coronal case, the structure of the algorithm is slightly different. As in the sagittal case, two starting positions are selected. Fig. 4c) demonstrates the different regions in the volume. The distances to the slices are the same as in the sagittal case, one fourth of the distance from the outermost part towards the outermost part of the other side. The same algorithm that is used to determine the start slices in the two other algorithms is here used for all slices from position $\mathrm{B}$ to $\mathrm{C}$ in conjunction with propagating information from previous slices, to avoid losing areas of the 
brain that are no longer connected to the largest region. For the slices from position A and position D, outwards, the same criteria as in the sagittal case are used. The information from previously segmented slices propagates throughout the traversing of the volume. An example of a segmented slice can be seen in Fig. 3d).

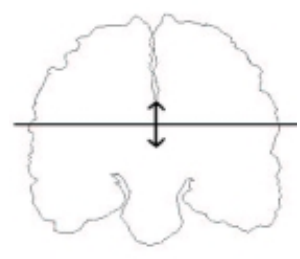

(a)

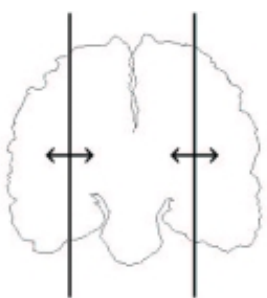

(b)

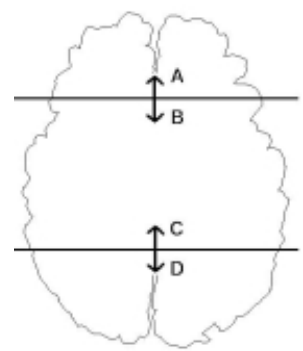

(c)

Fig. 4. How the algorithm advances on: a) A transaxial volume. b) A sagittal volume. c) A coronal volume.

\subsection{Segmentation algorithms}

The important steps of the algorithm are described using pseudo-code. First the thresholding process is described, then how the background is accounted for, and then how to create a start mask in the two cases depending on the orientation of the data. Lastly, it is described how the foreground, i.e. the brain tissue, is segmented. All binary dilations and erosions use a $3 \times 3$ structure element. Unless otherwise stated, operations on consecutive lines are performed in sequence using previous result.

\section{Histogram thresholding algorithm}

input: A volume of MR data of the brain, OrgImage.

A grey-level eroded volume, MinImage.

A grey-level dilated volume, MaxImage.

output: A mask with possible brain matter, OrgMatter.

A mask with darker brain matter, MinMatter.

A mask with possible fat, MaxFat.

using the histograms

generate continuous histogram from OrgImage

find 4 largest local max in 2nd derivative sort

select threshold between 2nd and 4th $\max \rightarrow$ OrgMatter

generate continuous histogram from MinImage

find 4 largest local max in 2nd derivative sort select threshold above 2nd max $\rightarrow$ MinMatter generate continuous histogram from MaxImage

find 4 largest local max in 2nd derivative sort select threshold above 3rd max $\rightarrow$ MaxFat end; 


\section{Background algorithm}

The algorithm is applied in two directions for maximum performance. If the data are sagittal, they are applied in the transaxial direction as well. For transaxial data, they are applied in the sagittal direction too. The results are then ORed together.

input: A mask with possible brain matter, OrgMatter.

A mask with possible fat, MaxFat.

output: A mask without brain matter, NoBrainMask.

on start slice

complement OrgMatter $\rightarrow$ OrgNoMatter

label MaxFat keep objects $>0.1 \%$ of brain size

MaxFat OR OrgNoMatter $\rightarrow$ NoBrainMask

traverse volume

dilate MaxFat

keep pixels under (dilate previous from NoBrainMask)

keep pixels under OrgMatter

OR complement OrgMatter

OR (label MaxFat keep objects $>5 \%$ of brain size) $\rightarrow$ NoBrainMask end;

\section{Start slice algorithm}

In transaxial data there is only need for one start slice, due to the anatomy of the brain. For the sagittal case there are two start slices generated in the middle of each hemisphere to avoid problems where the two brain halves meet. In the coronal case there are also two start slices to avoid difficulties when the brain is split up into several regions.

input: A mask with possible brain matter, OrgMatter.

A mask with darker brain matter, MinMatter.

A mask with possible fat, MaxFat.

output: A mask slice with the start slice of the brain, StartMask.

on start slice

complement OrgMatter OR MaxFat

erode MinMatter label keep largest region

dilate keep pixels under input mask fill holes

dilate keep pixels under OrgMatter fill holes store result in StartMask

end;

\section{Foreground algorithm}

On transaxial data the algorithm starts in a mid-brain slice and advances upwards and downwards. On sagittal data the algorithm starts at two positions, both in the middle of the right and the left hemispheres and advances to the left and to the right until they meet. On coronal data the algorithm starts at two positions in the brain (A and D) and advances out to the border of the brain, as can be seen in Fig. 4c). 
input: A mask with possible brain matter, OrgMatter.

A mask with darker brain matter, MinMatter.

A mask with non-brain matter, NoBrainMask.

A start mask StartMask

output: A mask with the segmented areas, Segm.

on start slice

store StartMask in Segm

fill holes $\rightarrow$ PrevSegm

traverse volume

select slice from MinMatter dilate fill holes

MinMatter AND PrevSegm dilate AND OrgMatter

AND NOT NoBrainMask $\rightarrow$ Segm

fill holes $\rightarrow$ PrevSegm

end;

Foreground algorithm coronal cases B and C

On coronal data the algorithm starts at two positions in the brain (B and $\mathrm{C}$ ) and advances into the centre of the brain, as can be seen in Fig. 4c). The algorithm is a combination of the start slice algorithm and the foreground algorithm.

input: A mask with possible brain matter, OrgMatter.

A mask with darker brain matter, MinMatter.

A mask with non-brain matter, NoBrainMask.

A start mask StartMask

output: A mask with the segmented areas, Segm.

on start slice

store StartMask in Segm

fill holes $\rightarrow$ PrevSegm traverse volume

Complement OrgMatter OR MaxFat

erode MinMatter label keep regions $>10 \%$ of brain size

dilate keep pixels under input mask fill holes

dilate keep pixels under OrgMatter fill holes

OrgMatter AND PrevSegm

dilate AND OrgMatter

AND NOT NoBrainMask $\rightarrow$ Segm

fill holes $\rightarrow$ PrevSegm

end;

\section{Results}

The algorithm has been tested on 30 sets of MRI data; for an example of how the data may look, see Fig. 5. The segmentation algorithm generates reproducible results and can therefore be used as a segmentation tool for visualisation purposes under clinical conditions. The algorithm has been visually evaluated on 30 patient data sets of transaxial, sagittal and coronal cases.

A problem that often occurs is that cranial nerves that link cerebral tissues to extra-cerebral nervous structures cause bridges that the binary morphology 


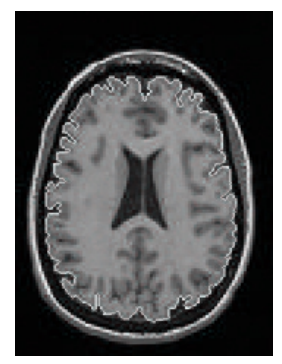

(a)

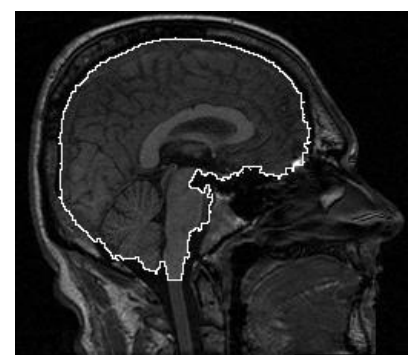

(a)

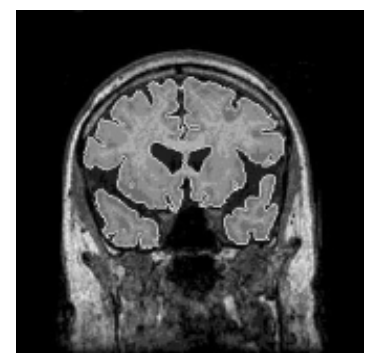

(c)

Fig. 5. Segmented slices. a) A transaxial slice. b) A sagittal slice. c) A coronal slice.

does not break. However, when involving both grey-level dilations and erosions, these bridges are almost always broken.

When a problem does arise it is possible to manually delete unwanted tissue on a mask and restart the algorithm from that particular slice. In some rare cases with extremely poor data, human interaction is necessary. In most MRI data-sets the quality is much better than the algorithm requires and the need for interaction is minimal.

\section{Future Work}

There will be further development of segmentation algorithms using grey-level morphology and also including artificial neural nets (ANN) from a program called BRAINS2 from the Mental Health Clinical Research Center, The University of Iowa College of Medicine and Hospitals and Clinics, Iowa City USA [8]. A large data base of coronal MRI scans is thus available at the HUBIN group. There will be sufficient cases to do a more thorough and statistically relevant testing of the methods developed.

\section{References}

1. R. C. Gonzalez and R. E. Woods. Digital Image Processing, chapter 8, pages 548-560. Addison-Wesley Publishing Company, Inc., 3 edition, 1992.

2. R. C. Gonzalez and R. E. Woods. Digital Image Processing, chapter 8, pages 550-560. Addison-Wesley Publishing Company, Inc., 2 edition, 2002.

3. R. Hult. Grey-level Morphology Based Segmentation of MRI of the Human Cortex. In Proceedings of ICIAP0111th International Conference on Image Analysis and Processing, Palermo, Italy, 2001.

4. R. Hult and E. Bengtsson. Combined Visualisation of Functional and Anatomical Brain Images. In Proceedings of $12^{\text {th }}$ Scandinavian Conference on Image Analysis, Bergen, Norway, 2001.

5. R. Hult, E. Bengtsson, and L. Thurfjell. Segmentation of the Brain in MRI Using Grey Level Morphology and Propagation of Information. In Proceedings of $11^{\text {th }}$ Scandinavian Conference on Image Analysis, Kangerlussuaq, Greenland, volume I, pages 367-373, 1999. 
6. D. H. Laidlaw, K. W. Fleischer, and A. H. Barr. Partial-Volume Baysian Classification of Material Mixtures in MR Volume Data Using Voxel Histograms. IEEE Transactions on Medical Imaging, 17(1):74-86, February 1998.

7. J. Lindblad. Histogram Thresholding using Kernel Density Estimates. In Proceedings of SSAB (Swedish Society for Automated Image Analysis) Symposium on Image Analysis, Halmstad, Sweden, pages 41-44, March 2000.

8. V. A. Magnotta, D. Heckel, N. C. Andreasen, T. Cizadlo, P. W. Corson, J. C. Ehrhardt, and W. T. Yuh. Measurement of brain structures with artificial neural networks: two- and three-dimensional applications. Radiology, 211(3):781-790, June 1999.

9. R. Momenan, D. H. ande Robert Rawlings, Ruttimann, M. Kerich, and D. Rio. Intensity-Adaptive Segmentation of Single-Echo T1-Weighted Magnetic Resonance Images. Human Brain Mapping, 5(3):194-205, 1997.

10. E. Parzen. On estimation of probability and mode. AMStat, 33:1065-1076, 1962.

11. M. Rosenblatt. Remarks on some nonparametric estimates of a density functione. AMStat, pages 642-669, 1956.

12. H. G. Schnack, H. E. H. Pol, W. F. C. Baaré, W. G. Staal, M. A. Viergever, , and R. S. Kahn. Automated Separation of Gray and White Matter from MR Images of the Human Brain. NeuroImage, 13(1):230-237, January 2001.

13. J. Serra. Image Analysis and Mathematical Morphology, volume 1. Academic Press, 1982.

14. J. Serra, editor. Image Analysis and Mathematical Morphology Volume 2: Theoretical Advances, volume 2. Academic Press, 1988.

15. R. Stokking, K. L. Vincken, and M. A. Viergever. Automatic Morphology-Based Brain Segmentation (MBRASE) from MRI-T1 Data . NeuroImage, 12(6):726-738, December 2000.

16. A. P. Zijdenbos, B. M. Dawant, R. a., and A. C. Palmer. Morphometric Analysis of White Matter Lesions in MR Images: Method and Validation. IEEE Transactions on Medical Imaging, 13(4):716-724, December 1994.

17. M. Özkan, B. M. Dawant, and R. J. Maciunas. Neural-Network-Based Segmentation of Multi-Modal Medical Images: A Comparative and Prospective Study. IEEE Transactions on Medical Imaging, 12(3):534-544, September 1993. 